\title{
ASYMPTOTIC ANALYSIS OF THE CLOSED NETWORK WITH TIME-DEPENDENT SERVICE PARAMETERS AND SINGLE-TYPE MESSAGES
}

\author{
Mikhail Matalytski ${ }^{1}$, Tatiana Rusilko ${ }^{2}$, Andrey Pankov ${ }^{2}$ \\ ${ }^{1}$ Institute of Mathematics, Czestochowa University of Technology, Czestochowa, Poland \\ ${ }^{2}$ Grodno State University, Grodno, Belarus \\ m.matalytski@gmail.com,romaniuk@grsu.by,a.pankov@gmail.com
}

\begin{abstract}
A queueing network of any structure with single-type messages is considered in the paper. An asymptotic analysis of the network in case of large number of service requests conducted. It is suggested that the service parameters of each queueing system of the network, as well as the probability of messages transition between systems, depend on time. A system of ordinary differential equations to calculate the average relative number of messages in each queueing system, depending on the time, was obtained. There is one calculated example in the article.
\end{abstract}

\section{Introduction}

Currently, much attention is paid to the mathematical modeling of various technical, economic and other processes and objects. Mathematical models allow one to study the properties and behavior of real simulated objects, without the practical experience that it is often impossible or impractical to carry out. Recently, one of the most frequently used probabilistic models related in time, linking the functioning of many disparate systems are queuing networks (QN).

The development of new approaches and methods of modern QN theory have often dictated the need to consider a number of features of the simulated object. For example, the QN are used as models of the processing of customer claims for an insurance company [1-4]. However, the parameters of customer service in the insurance companies are not constant throughout the period of time. In this regard, there is a problem to study of queueing networks provided depending on time of the intensities of service requests, the number of service lines and transition probabilities between the systems of the network.

\section{Diffusion approximation of queueing network of any structure with single-type messages}

Consider a closed queueing network consisting of $n+1$ queueing systems (QS) $S_{0}, S_{1}, \ldots, S_{n}$, where the total number of single-type messages equals $K$. Suppose 
that the parameters of services of this network depend on time $t, t \in[0, T]$. Let the number of service lines in the system $S_{i}$ at time $t$ is described by a function of time $m_{i}(t)$, that takes integer values, $i=\overline{0, n}$. The service time in each of the lines of $S_{i}$ is distributed exponentially with time-dependent mean value $\mu_{i}^{-1}(t)$, and $\mu_{i}(t) \geq 0, i=\overline{0, n}$. Requests for service are selected according to the discipline FIFO. Message that completed servicing in the system $S_{i}$, with the probability of $p_{i j}(t)$ goes to the queue of system $S_{j}, i, j=\overline{0, n}$. The transition matrix $P(t)=\left\|p_{i j}(t)\right\|$ is the matrix of transition probabilities of an irreducible Markov chain, and generally depends on the time, $0 \leq p_{i j}(t) \leq 1, \sum_{j=0}^{n} p_{i j}(t)=1$. The main objective of the study of QN described here is the asymptotic analysis of the Markov process describing its behavior, with a large number of messages. The state of the network at time $t$ is described by the vector

$$
k(t)=\left(k_{1}(t), k_{2}(t), \ldots, k_{n}(t)\right),
$$

where $k_{i}(t)$ - the number of messages in the system $S_{i}$ at time $t, t \in[0, T]$, $i=\overline{1, n}$, which forms the $n$-dimensional Markov process with continuous time and finite number of states. Because the network is closed, it is obvious that the number of messages in the system $S_{0}$ is equal to $k_{0}(t)=K-\sum_{i=1}^{n} k_{i}(t)$.

Theorem. Probability density $p(x, t)$ of vector of relative variables $\xi(t)=\left(\frac{k_{1}(t)}{K}, \frac{k_{2}(t)}{K}, \ldots, \frac{k_{n}(t)}{K}\right)$, provided that it is differentiable on $t$ and twice piecewise continuously differentiable in $x_{i}, i=\overline{1, n}$, satisfies up to $\mathrm{O}\left(\varepsilon^{2}\right)$, where $\varepsilon=\frac{1}{K}$, Kolmogorov-Fokker-Planck equation

$$
\frac{\partial p(x, t)}{\partial t}=-\sum_{i=1}^{n} \frac{\partial}{\partial x_{i}}\left(A_{i}(x, t) p(x, t)\right)+\frac{\varepsilon}{2} \sum_{i, j=1}^{n} \frac{\partial^{2}}{\partial x_{i} \partial x_{j}}\left(B_{i j}(x, t) p(x, t)\right)
$$

where

$$
\begin{aligned}
& A_{i}(x, t)=\sum_{j=0}^{n} \mu_{j}(t) p_{j i}^{*}(t) \min \left(l_{j}(t), x_{j}\right), \\
& B_{i i}(x, t)=\sum_{j=0}^{n} \mu_{j}(t) r_{j i}(t) \min \left(l_{j}(t), x_{j}\right),
\end{aligned}
$$




$$
\begin{gathered}
B_{i j}(x, t)=-\mu_{i}(t) p_{i j}(t) \min \left(l_{i}(t), x_{i}\right), \\
p_{j i}^{*}(t)=r_{j i}(t)=p_{j i}(t), \quad i \neq j ; p_{i i}^{*}(t)=-r_{i i}(t)=-1+p_{i i}(t), \quad i=j .
\end{gathered}
$$

Proof. Consider all the possible transitions in the state $k(t+\Delta t)=(k, t+\Delta t)$ of the process in the time $\Delta t$ :

- from state $\left(k+I_{i}-I_{j}, t\right)$ can get into $(k, t+\Delta t)$ with probability

$$
\mu_{i}(t) \min \left(m_{i}(t), k_{i}(t)+1\right) p_{i j}(t) \Delta t+\mathrm{o}(\Delta t), i, j=\overline{0, n} ;
$$

- from state $(k, t)$ - with probability

$$
1-\sum_{i=0}^{n} \mu_{i}(t) \min \left(m_{i}(t), k_{i}(t)\right) \Delta t+\mathrm{o}(\Delta t)
$$

- from other states - with probability $\mathrm{o}(\Delta t)$. Here $I_{i}$ - $n$-dimensional zerovector, with acceptance of $i$-th component equals 1 , when $1 \leq i \leq n, I_{0}$ $n$-dimensional zero-vector.

Applying the formula of total probability, we can write the system of differential equations for the state probabilities $P(k, t)$ :

$$
\begin{gathered}
P(k, t)=\sum_{i, j=0}^{n} \mu_{i}(t) p_{i j}(t) \min \left(m_{i}(t), k_{i}(t)+1\right) P\left(k+I_{i}-I_{j}, t\right) \Delta t+ \\
\quad+\left(1-\sum_{i, j=0}^{n} \mu_{i}(t) p_{i j}(t) \min \left(m_{i}(t), k_{i}(t)\right) \Delta t\right) P(k, t)+\mathrm{o}(\Delta t) .
\end{gathered}
$$

Using the limit for $\Delta t \rightarrow 0$, we obtain a system of difference-differential equations for the probability of Kolmogorov states:

$$
\begin{gathered}
\frac{d P(k, t)}{d t}=\sum_{i, j=0}^{n} \mu_{i}(t) p_{i j}(t) \min \left(m_{i}(t), k_{i}(t)\right)\left(P\left(k+I_{i}-I_{j}, t\right)-P(k, t)\right)+ \\
+\sum_{i, j=0}^{n}\left[\mu_{i}(t) p_{i j}(t)\left(\min \left(m_{i}(t), k_{i}(t)+1\right)-\min \left(m_{i}(t), k_{i}(t)\right)\right) P\left(k+I_{i}-I_{j}, t\right) .\right.
\end{gathered}
$$

Next, we consider the case of a large number of messages in the network, $K \gg>1$, and move to the vector of relative variables $\xi(t)=\left(\frac{k(t)}{K}\right)$, the possible values of which belong to the bounded closed set $G=\left\{x=\left(x_{1}, x_{2}, \ldots, x_{n}\right): x_{i} \geq 0, i=\overline{1, n}, \sum_{i=1}^{n} x_{i} \leq 1\right\}$, in which they are located at the nodes of $n$-dimensional lattice at a distance $\varepsilon=\frac{1}{K}$ 
from each other. With increasing $K$ "filling density" of the set $G$ by possible components of this vector increases, and it becomes possible to believe that he has a continuous distribution with the probability density $p(x, t)=K^{n} P(x K, t), x \in G$, where $p(x, t)$ is the meaning of the probability density function of the random vector $\xi(t)$.

Denoted by $e_{i}-n$-dimensional zero-vector, with exceptance of $i$-th component equals $\varepsilon, i=\overline{1, n}, c(u)=\left\{\begin{array}{l}1, u>0, \\ 0, u \leq 0\end{array}\right.$. Note that $\min (u, v+1)=\min (u, v)+c(u-v)$, $c(u-v)=\frac{\partial \min (u, v)}{\partial v}$, because $\min (u, v)=\left\{\begin{array}{l}v, u \geq v, \\ u, u<v\end{array}\right.$. Introduce the notation $l_{i}(t)=\frac{m_{i}(t)}{K}, i=\overline{1, n}$. Rewriting the system of equations (2) for density $p(x, t)$, we obtain

$$
\begin{aligned}
\frac{\partial p(x, t)}{\partial t} & =\sum_{i, j=0}^{n} K \mu_{i} p_{i j}(t) \min \left(l_{i}(t), x_{i}\right)\left(p\left(x+e_{i}-e_{j}, t\right)-p(x, t)\right)+ \\
& +\sum_{i, j=0}^{n} \mu_{i}(t) p_{i j}(t) \frac{\partial \min \left(l_{i}(t), x_{i}\right)}{\partial x_{i}} p\left(x+e_{i}-e_{j}, t\right)
\end{aligned}
$$

We represent the right-hand side of this system, up to terms of order of smallness $\varepsilon^{2}$. We will assume that $p(x, t)$ is differentiable both at $t$ and twice continuously differentiable at $x_{i}, i=\overline{1, n}, \frac{\partial p(x, t)}{\partial x_{0}}=0$. Then the following Taylor series expansion takes place:

$$
\begin{gathered}
p\left(x+e_{i}-e_{j}, t\right)=p(x, t)+\varepsilon\left(\frac{\partial p(x, t)}{\partial x_{i}}-\frac{\partial p(x, t)}{\partial x_{j}}\right)+ \\
+\frac{\varepsilon^{2}}{2}\left(\frac{\partial^{2} p(x, t)}{\partial x_{i}^{2}}-2 \frac{\partial^{2} p(x, t)}{\partial x_{i} \partial x_{j}}+\frac{\partial^{2} p(x, t)}{\partial x_{j}^{2}}\right)+o\left(\varepsilon^{2}\right), i, j=\overline{1, n} .
\end{gathered}
$$

Using the last expansion and the fact that $\varepsilon K=1$, we obtain:

$$
\begin{aligned}
\frac{\partial p(x, t)}{\partial t} & =\sum_{i, j=0}^{n} \mu_{i}(t) p_{i j}(t) \min \left(l_{i}(t), x_{i}\right)\left[\left(\frac{\partial p(x, t)}{\partial x_{i}}-\frac{\partial p(x, t)}{\partial x_{j}}\right)+\right. \\
& \left.+\frac{\varepsilon}{2}\left(\frac{\partial^{2} p(x, t)}{\partial x_{i}^{2}}-2 \frac{\partial^{2} p(x, t)}{\partial x_{i} \partial x_{j}}+\frac{\partial^{2} p(x, t)}{\partial x_{j}^{2}}\right)\right]+
\end{aligned}
$$




$$
\begin{gathered}
+\sum_{i, j=0}^{n} \mu_{i}(t) p_{i j}(t) \frac{\partial \min \left(l_{i}(t), x_{i}\right)}{\partial x_{i}}\left[p(x, t)+\varepsilon\left(\frac{\partial p(x, t)}{\partial x_{i}}-\frac{\partial p(x, t)}{\partial x_{j}}\right)+\right. \\
\left.+\frac{\varepsilon^{2}}{2}\left(\frac{\partial^{2} p(x, t)}{\partial x_{i}^{2}}-2 \frac{\partial^{2} p(x, t)}{\partial x_{i} \partial x_{j}}+\frac{\partial^{2} p(x, t)}{\partial x_{j}^{2}}\right)\right]+o\left(\varepsilon^{2}\right) . .
\end{gathered}
$$

Using the notation (3), (4), this equation can be written as (2) up to terms of order of smallness $\varepsilon^{2}$.

\section{Calculation of the average relative number of messages in each systems of the QN}

Equation (2) is the equation of the Kolmogorov-Fokker-Planck equation for the probability distribution of the Markov process $\xi(t)$. Therefore, the components of the average relative number of messages in the systems of the QN $n(t)=\left(n_{1}(t), n_{2}(t), \ldots, n_{n}(t)\right)$, where $n_{i}(t)=M\left(\xi_{i}(t)\right)=M\left(\frac{k_{i}(t)}{K}\right)$, according to $[2,3]$, up to terms of order of smallness $O\left(\varepsilon^{2}\right)$ satisfy the system of ordinary differential equations

$$
n_{i}^{\prime}(t)=A_{i}(n(t)), i=\overline{1, n}
$$

Using (3), system (5) is:

$$
n_{i}^{\prime}(t)=\sum_{j=0}^{n} \mu_{j}(t) p_{j i}^{*}(t) \min \left(l_{j}(t), n_{j}(t)\right) .
$$

The right-hand side of (6) are continuous piecewise linear functions. These systems will be solved by splitting the phase space and solving in the areas of linearity of their right-hand sides. We define an explicit form of these equations in the areas of linear theirity of their right-hand sides. Let $\Omega(t)=\{0,1,2, \ldots, n\}$ - the set of components indices of vector $n(t)$. We divide $\Omega(t)$ into two disjoint sets $\Omega_{0}(t)$ and $\Omega_{1}(t)$ :

$$
\Omega_{0}(t)=\left\{i: l_{i}(t)<n_{i}(t) \leq 1\right\}, \Omega_{1}(t)=\left\{j: 0 \leq n_{j}(t) \leq l_{j}(t)\right\} .
$$

For a fixed $t$ number of partitions of this type is equal to $2^{n+1}$. Each partition on set $G(t)=\left\{n(t): n_{i}(t) \geq 0, \sum_{i=0}^{n} n_{i}(t)=1\right\}$ will define disjoint sets $G_{\tau}(t)$ such that 


$$
\begin{gathered}
G_{\tau}(t)=\left\{n(t): l_{i}(t)<n_{i}(t) \leq 1, i \in \Omega_{0}(t) ; 0 \leq n_{j}(t) \leq l_{j}(t), j \in \Omega_{1}(t) ; \sum_{c=0}^{n} n_{c}(t)=1\right\}, \\
\tau=1,2, \ldots, 2^{n+1}, \bigcup_{\tau=1}^{2^{n+1}} G_{\tau}(t)=G(t) .
\end{gathered}
$$

Now we can write the system of equations (6) explicitly for each region $G_{\tau}(t)$. For instance, in region

$$
A: \Omega_{1}(t)=\{1,2, \ldots, n\}, \Omega_{0}(t)=\{0\},
$$

which corresponds to empty queue in average in QS $S_{i}, i=\overline{1, n}$, we obtain the following system of differential equations:

$$
n_{i}^{\prime}(t)=\mu_{0}(t) p_{0 i}^{*}(t) l_{0}(t)+\sum_{j=1}^{n} \mu_{j}(t) p_{j i}^{*}(t) n_{j}(t) .
$$

The solution of (7) for certain initial conditions can find the average relative number $n_{i}(t)$, and hence the average number $K n_{i}(t)$, of messages in each of the QS of the network.

\section{Example}

Consider a QN, consisting of four QS $S_{0}, S_{1}, S_{2}, S_{3}$, in which circulates $K=30000$ single-type messages. We define the following probabilities of transitions between the queueing systems of the network:

$$
\begin{gathered}
p_{03}(t)=1-p_{01}(t), p_{10}(t)=1-p_{12}(t), \\
p_{21}(t)=1-p_{23}(t), p_{32}(t)=1-p_{30}(t), \\
p_{01}(t)=p_{12}(t)=0.4(\cos (t)+1), \\
p_{23}(t)=0.4(\sin (t)+1), \\
p_{30}(t)=0.2(\sin (t)+1), \\
p_{i j}(t)=0 \text { in other case. }
\end{gathered}
$$

Suppose that at the initial time all messages are concentrated in the system $S_{0}$, i.e. $n_{i}(0)=0, i=\overline{1,3}$, and on the interval of time, in average there are no queues in systems $S_{i}, i=\overline{1,3}$. System (7) in this case would be: 


$$
\left\{\begin{array}{l}
n_{1}^{\prime}(t)=\mu_{0}(t) p_{01}(t) l_{0}(t)+\mu_{2}(t) p_{21}(t) n_{2}(t)-\mu_{1}(t) n_{1}(t), \\
n_{2}^{\prime}(t)=\mu_{1}(t) p_{12}(t) n_{1}(t)+\mu_{3}(t) p_{32}(t) n_{3}(t)-\mu_{2}(t) n_{2}(t), \\
n_{3}^{\prime}(t)=\mu_{0}(t) p_{03}(t) l_{0}(t)+\mu_{2}(t) p_{23}(t) n_{2}(t)-\mu_{3}(t) n_{3}(t) .
\end{array}\right.
$$

Obviously, if $\mu_{i}(t) p_{i j}(t) \min \left(l_{i}(t), n_{j}(t)\right)$ is the flow of the average relative number of messages from system $S_{i}$ to system $S_{j}$, the system of differential equations (8) corresponds to the well-known rule: the derivative of the average relative number of messages in each of the systems on the network is the sum of all flows of the mean relative number of messages, incoming into this system minus the sum of all flows outcoming from the system.

The intensity of service requests in systems of the network will be given as the following functions:

$$
\begin{gathered}
\mu_{1}(t)=0.1+0.5 t, \quad \mu_{2}(t)=0.33+0.5 t \\
\mu_{3}(t)=0.55+0.05 t, \quad \mu_{0}(t)=20+0.05 t
\end{gathered}
$$

Let the number of service lines in the system $S_{0}$ be set equal to

$$
m_{0}(t)=[5 \sin (5 t)+10] \text {, where [·] - integer part of the number. }
$$

System (8) is a system of linear differential equations with non-constant coefficients. The analytical solution of this system is difficult. For the numerical solution of differential equations of the form (8) mathematical computer system Maple can be applied.

Figure 1 shows graphically the behavior of $n_{2}(t)$ as a function of time. Similarly, remaining components of vector $n(t)$ can be examined, which are the solution of (8).

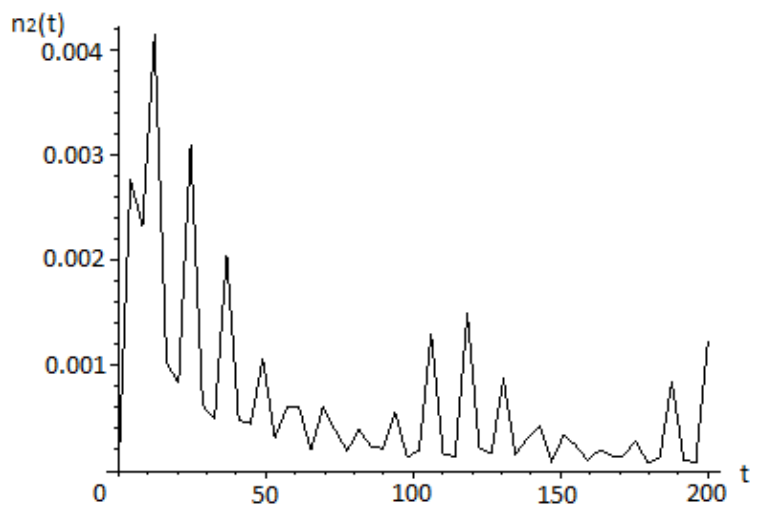

Fig. 1. Graph of $n_{2}(t)$ 


\section{Conclusions}

The considered method of calculating of the average relative number of messages in systems of queueing network is valid only for heavily loaded networks, that is, in the case of a large number of messages $K$. The accuracy of the method increases with the number of messages in the network.

\section{References}

[1] Matalytski M., Rusilko T., Mathematical analysis of stochastic models of claims processing in insurance companies, GrSU, Grodno 2007.

[2] Medvedev G., On the optimization of closed queuing system, Proceedings of the AS USSR, Technical Cybernetics 1975, 6, 65-73.

[3] Paraev U., Introduction in Statistical Dynamics of Control and Filtration Processes, Sovetskoe Radio, Moscow 1976.

[4] Rusilko T., Investigation of stochastic model of customer claims processing in the insurance company with a time-dependent parameters of service, Vesnik GrSU 2011, 2(3), 167-174. 